\title{
Evaluation of changes in sleep breathing patterns after primary palatoplasty in cleft children
}

\author{
Reilly, Justice E. ; Russel, CraigJ. H.*; Gibson, Neil A. ; Moores Tony*; Ray, Arup K. *; Devlin, Mark F.; Wynne, David M. * \\ * Royal Hospital for Sick Children, Yorkhill, Glasgow, Scotland
}

\begin{abstract}
Introduction: There is a need to more clearly understand the characteristics of breathing patterns in children with cleft palate in the first year of life, as there is little data available to guide current practice. Pierre Robin patients are known to have a higher incidence, however we hypothesised sleep breathing disturbance is not confined to this sub-group of cleft patient.

Methods: We conducted a prospective observational study of sleep disordered breathing patterns in a cohort of infants with oronasal clefts (cleft palate with or without cleft lip) to describe the spectrum of sleep breathing patterns both pre and post palate repair. Sleep breathing studies were performed pre- and post-operatively in sequential infants referred to a regional cleft lip and palate unit. Results of sleep breathing studies were analysed according to American Academy of Sleep Medicine scoring guidelines and correlated with clinical history and details of peri-operative respiratory compromise. The degree of sleep disordered breathing was characterised using desaturation indices (number of desaturations from baseline $\mathrm{SpO}_{2}$ of $>=4 \%$, per hour).

Results: Thirty-nine infants were included in this study, twenty-five female and fourteen male. Twelve had isolated Cleft Palate as part of an associated syndrome. Patients were categorised into Isolated Cleft Palate, Isolated Cleft Palate in the context of Pierre Robin Sequence, and those with Cleft Lip and Palate. All groups demonstrated some degree of sleep breathing abnormality. Not unsurprisingly the eight infants with Pierre Robin Sequence had a significantly higher desaturation index before surgical intervention $(\mathrm{p}=0.043)$, and were more likely to require a pre-operative airway intervention $(\mathrm{p}=0.009)$. Palate repair in this group did not alter the relative distribution of patients in each severity category of sleep disorder breathing. Surgical repair of the secondary palate in the remaining children was associated with some improvement but by no means complete resolution of their sleep disordered breathing patterns.

Conclusions: We conclude that sleep breathing disturbance is not confined to Pierre Robin patients alone and all cleft palate patients should undergo pre-operative and post-operative sleep breathing analysis.
\end{abstract}

Reilly JE, Russel CJH, Gibson NA, Moores T, Ray AK, Devlin MF, Wynne DM. Evaluation of changes in sleep breathing patterns after primary palatoplasty in cleft children. South Eur J Orthod Dentofac Res. 2014; 1:10-14.

Submitted: November1, 2013. ; Accepted: February 15, 2014. ; Published: April 25, 2014

\section{INTRODUCTION}

Cleft Lip and/or Palate (CL/P) is one of the most common congenital craniofacial anomalies with an overall incidence of around 1 in 700 live births in the UK. ${ }^{1}$ However this figure changes from between 1 in 400 to 1 in 2500 dependent on the ethno-geographic population studied. ${ }^{2}$ Patients with clefts that affect the secondary palate can present as either cleft lip and palate (CLP), or isolated cleft palate (CP).

Corresponding Author:

David M. Wynne

Department of Paediatric Otorhinolaryngology, The Royal

Hospital For Sick Children, Yorkhill, Glasgow, Scotland G3 8SJ

e-mail: David.Wynne@ggc.scot.nhs.uk
CLP patients can be either unilateral or bilateral while any component can be either complete or incomplete. Overall approximately $70 \%$ of cleft lip or palate presentations occur as an isolated defect. The remaining $30 \%$ occur in the context of a syndrome or chromosomal abnormality. ${ }^{3}$ Children with cleft palate with or without cleft lip $(\mathrm{CP} \pm \mathrm{L})$ have a potential increased risk of sleep disordered breathing (SDB) due to increased up per airway resistance secondary to alterations in nasopharyngeal anatomy and physiology. ${ }^{4}$ There is a need to more clearly understand the range and pattern of sleep breathing disturbance in $\mathrm{CP} \pm \mathrm{L}$. In older children $\mathrm{SDB}$ is known to be associated with a range of clinical problems. These include neuro-behavioural difficulties, daytime somnolence and poor school performance. 
In adults SDB increases serum markers known to be associate with the development of atherosclerosis. ${ }^{5,6}$ It is speculated that these effects occur through mechanisms mediated by intermittent hypoxia and associated fluctuations in autonomic sympathetic tone. The risk of such problems with SDB in infancy is unknown. In addition operative repair of $\mathrm{CP}$ deformities has the potential to increase the risk of upper airway obstruction as closure of an open cleft reduces nasopharyngeal dimensions. ${ }^{7}$

The Gold Standard investigation for sleep disordered breathing is laboratory polysomnography (PSG) which may include electroencephalogram leads, electrooculogram, electromyogram, nasal pressure/oral thermistor, electrocardiogram, pulse oximetry, chest and abdominal excursion belts, plethysmography, limb leads, end-tidal or transcutaneous $\mathrm{CO} 2$, oesophagealmanometry, and audiovisual taping. ${ }^{8}$ PSG is expensive and requires facilities beyond the reach of many units, even in high-income countries. Commonly, alternative investigations are employed with good sensitivity including overnight pulse oximetry and transcutaneous oxygen saturation and carbon dioxide tension (TOSCA) studies. ${ }^{9}$ To more clearly understand this issue that is poorly characterised in the current literature, we looked at all sleep studies performed in cleft children treated at our institution over the period of a year. Pre- and postoperative sleep study results were analysed in relation to infants' clinical presentation and evidence of pre- and postoperative respiratory compromise.

\section{MATERIALS AND METHODS}

The West of Scotland Cleft service provides multidisciplinary care for patients from the pre-natal period into adolescence and covers a wide geographical area. All consecutive patients with cleft of the secondary palate undergoing primary repair in 2010 were included. Sleep studies were arranged according to physical resource availability and patient clinical characteristics. Type of sleep study used depended on the patient's geographical distance from the Royal Hospital for Sick Children and need for inpatient stay for other reasons. Sleep studies employed therefore ranged from overnight oxygen saturation monitoring using the $\mathrm{Masimo}^{\circ}$ system [Masimo Corporation, California USA] through to TOSCA monitoring and PSG studies, using the SomnoMed ${ }^{\circ}$ system [SomnoMed Limited, New South Wales, Australia] and reported using the American Academy of Sleep Medicine scoring guidelines. Patients with submucous clefts were excluded as they tended to present later in childhood and as such are not comparable to the population of interest. Prestudy preconceptions and resource limitations also resulted in exclusion of isolated cleft lip patients.

Thirty-nine unselected consecutive patients, fourteen male and twenty-five female, treated between January 2010 and January 2011 were invited to take part in the study. Demographic data including cleft characteristics and age at cleft repair was collected for all thirty-nine patients, including their need for pre- and postoperative respiratory support.
From the age of three months patients were offered preoperative sleep studies. The average age at cleft repair was 9.2 months. Seven patients did not attend for pre-operative sleep study despite three offers of appointment. These patients all proceeded to cleft repair within our routine time frame. The sleep study date for patients with 'syndromes' other than Pierre Robin Sequence (PRS) ( $\mathrm{n}=4$, two pre-op and two postop patients) were excluded from further analysis due to small numbers, $(n=1)$ for each of the syndromes identified.

Intravelar veloplasty was the usual method of cleft palate repair. One patient was documented as having a Furlow palatoplasty for a narrow cleft.

All patients were offered PSG, however due to the wide geographic spread of the patient population across the highlands and islands of Western Scotland a number of other more pragmatic investigations including TOSCA and Masimo $^{\circ}$ overnight oximetry were also employed. Severity of SDB were determined by Desaturation Index (DI), a measure common to all three investigations utilized. Desaturation events were recorded as drops greater than or equal to $4 \%$ from baseline oxygen saturation as this is the standard used in oximetry literature. Beyond the neonatal period 4-5 desaturations per hour are considered within the normal range. Extrapolating from PSG data on breathing events we defined a desaturation index of 5-10 per hour would be considered a mild elevation and $>10$ as moderate to severe.

All patients undergoing cleft palate repair in our unit are routinely admitted to a High Dependency Unit (HDU) for close nursing observation, but not routine respiratory support. This is an issue of local practicalities and is not routine practice across the UK. We therefore also evaluated the need for pre-operative airway adjuncts and post-operative High Dependency Unit (HDU) respiratory support.

For the purposes of analysis patients were grouped according to cleft type. Cleft lip and palate is most commonly nonsyndromic and thought to have a distinct pathogenesis from Cleft Palate. We chose to look at non-syndromic patients to allow the investigation of the effect of cleft palate without potential confounding influence. Patients with PRS were seen as a separate group given the widely held recognition of their obstructive breathing issues.

\section{RESULTS}

Thirty-nine patients underwent primary palate repair in the allocated time period (Table 1). Thirty-two patients attended for preoperative sleep studies, two of whom had syndromes other than PRS and were excluded from further analysis. Twenty (66\%) had PSG studies, eight (27\%) had a TOSCA study and two (7\%) had an oximetry study preoperatively. Twenty-one patients had postoperative sleep studies, two of which were excluded from analysis as they were done on non-PRS, syndromic patients. Of the nineteen evaluated, thirteen (68\%) had PSG studies, three (16\%) had TOSCA studies and three (16\%) had oximetry studies. Sleep studies were carried out on average 49 days preoperatively 
and 110 days postoperatively. Sixteen patients had both pre- and postoperative studies, seven of whom had pre and postoperative PSG studies.

Figure 1 demonstrates the distribution of desaturation indices by cleft type prior to, and following surgical intervention. The extent of SDB, as described by DI, is significantly greater in the Pierre Robin Sequence (PRS) population when compared to non-syndromic isolated CP patients preoperatively, $(\mathrm{p}=0.043$, One Sided Wilcoxon Rank Sum Test). The preoperative desaturation indices (median and interquartile range (IQR)) for these three groups were 1.93 (0.7-3) (U+BCLP) / 3.53 (2.3-6.7) (Non Syndromic, Isolated CP) / 6.12 (1.91-25.9) (PRS).

The range of desaturation frequency is significantly wider for the PRS group ( $\mathrm{p}=0.043)$. The postoperative DI (median and IQR) for the same patient groups were $2.8(1.2-4.7)$ $(\mathrm{U}+\mathrm{BCLP}) / 1.6$ (1.5-2.3) (Non Syndromic, Isolated CP) / 5.72 (1.8-13.5) (PRS), with no significant difference between the groups.

Perhaps unsurprisingly PRS patients require significantly greater pre-operative respiratory intervention with either nasopharyngeal airway (NPA) or tracheostomy when compared with all other patients in the study, excluding syndromic CP (Fisher's Exact Test $\mathrm{p}=0.0026,4$ of 8 PRS patients versus 0 of 22 other cleft types).

Figure 2 shows the percentage of patients within each cleft presentation by the relative severity of their SDB. Desaturation Index data are presented as percentages of the total number of each cleft diagnosis to allow direct comparison. The desaturation events are shown on PSG to be most commonly due to airway obstruction. Repair of the secondary palate is associated with a small to moderate improvement in the distribution of desaturation indices in non-syndromic isolated cleft palate and cleft lip and palate patients. However the distribution of desaturation indices in PRS patients remains essentially unchanged following palate repair.

We reviewed the postoperative data from patients undergoing palate repair.

Table 1. Table describing all patients in terms of sex, cleft type and associated syndrome

\begin{tabular}{lcc}
\multicolumn{1}{c}{ Cleft Type } & Male & Female \\
\hline & $\mathrm{n}=14$ & $\mathrm{n}=25$ \\
\hline Pierre Robin Sequence & 2 & 6 \\
\hline Syndromic, Non-Pierre Robin CP ${ }^{*}$ & 1 & 3 \\
\hline Non-Syndromic CP & 3 & 10 \\
\hline Non-Syndromic CLP & 8 & 6 \\
\hline Unilateral CLP & 5 & 3 \\
\hline Bilateral CLP & 3 & 3 \\
\hline
\end{tabular}

* The male syndromic patient had Foetal Alcohol Syndrome with Micrognathia. The three female syndromic patients had Stickler Syndrome, Treacher Collins Syndrome and Significant Growth Retardation with Bilateral Triphalangeal Thumbs.
Figure 1. Distribution of Desaturation Indices by Cleft Type

The inter-quartile and full range of post-operative desaturation indices for each cleft presentation studied is demonstrated below. For the purposes of calculating the Desaturation Index (DI), desaturations were regarded as drops in $\mathrm{SaO} 2$ of $>4 \%$ from baseline oxygen saturation. Data from nonPRS, syndromic patients were excluded from these graphs.

\section{$U+B C L P=$ Unilateral and Bilateral Cleft Lip and Palate}

$P R S=$ Cleft Palate with Pierre Robin Syndrome

$C P=$ Isolated Cleft Palate

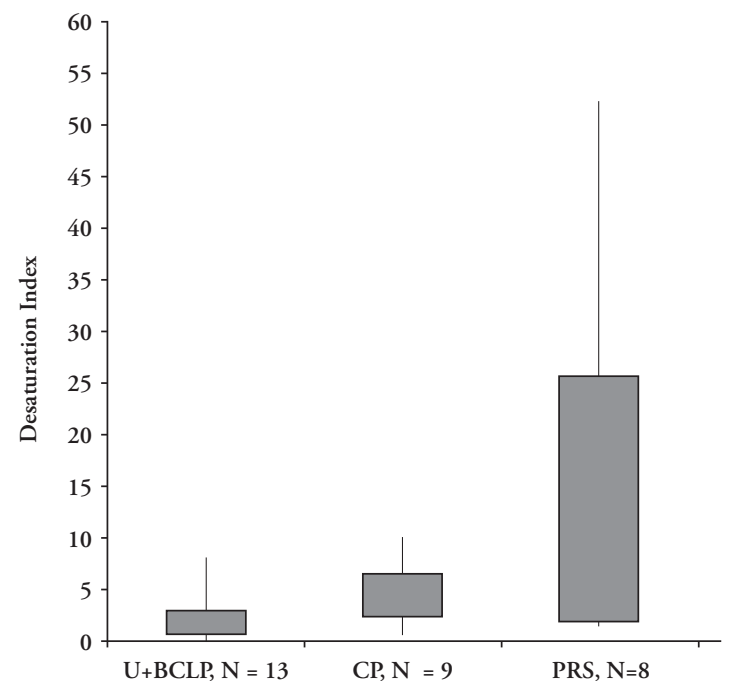

\section{1a. Preoperative Desaturation Indices}

The median desaturation index for each group is as follow; $U+B C L P: 1.93$, CP: 3.53 and PRS: 6.12. One-sided Wilcoxon rank sum test demonstrated a significant difference in desaturation indices between the non-syndromic cleft palate patients and those with the Pierre Robin Sequence $(p=0.043)$.

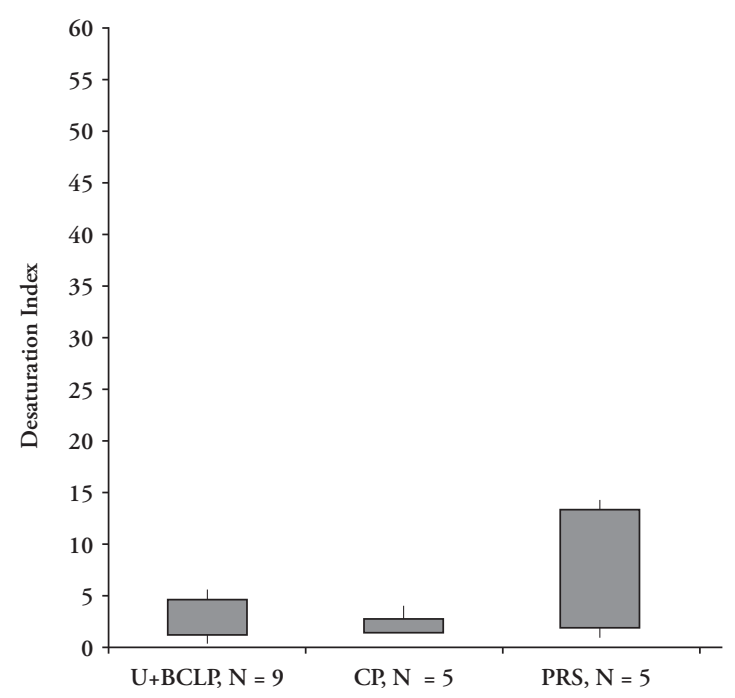

\section{1b. Postoperative Desaturation Indices}

The median desaturation index for each group is as follows; U+BCLP: 2.8, CP: 1.6 and PRS: 5.72. There was no significant difference between Desaturation Indices in these groups. 
These patients were all routinely taken to the HDU postoperatively for close saturation monitoring as is our routine practice. Again not unsurprisingly (and irrespective of whether pre or postoperative sleep study was performed) PRS patients are at greater risk of postoperative respiratory compromise (requirement for NPA insertion) when compared to all other patients analyzed (Fisher's Exact Test $\mathrm{p}=0.002,5$ of 8 PRS patients versus 0 of 27 other cleft types).

\section{DISCUSSION}

These data have demonstrated a wide range of pre and postoperative SDB in infants with $\mathrm{CP}+/-\mathrm{L}$. Virtually all the children studied in this report had evidence of some degree of disturbance in their sleep breathing pattern. Surgical repair of the secondary palate in non-PRS isolated cleft palate children and cleft lip and palate children was associated with some improvement but by no means complete resolution of their SDB. Pre-operative data presented here are in general agreement with the results of a similar study recently published from Australia. ${ }^{4}$ Unfortunately this study did not investigate the effect of surgery on SDB patterns.

The present study is limited by a relatively small cohort size of thirty-five patients after exclusions. This number represents all thirty nine patients undergoing cleft repair within the time frame, but excludes four patients who had different syndromes other than PRS as their sleep study data were not analysed. Institution resource pressures resulted in only sixteen patients having both pre- and post-operative sleep studies. Given that this study was not blinded, the desaturation indices were measured by the saturation monitors and interpreted by a Respiratory Physiologist and again by a Respiratory Physician. Examiners were not blinded as to the patient group, in fact on the request form for the sleep study, they were told the indication (i.e. preoperative / post operative sleep study) for the study. Clinician bias may have influenced those patients who attended for post-operative PSG given the known pre-operative study results and patient need for pre/ post-operative airway interventions. Our ability to perform accurate paired analysis of sleep studies was therefore limited due to sleep study discrepancy and also because patients with notable SDB had airway intervention which improved their breathing physiology. There was disappointing uptake of postoperative sleep studies with only twenty-one of invited patients attending. The nature of the geographic area (extremes of distance and island life) covered by our service meant that attendance for overnight in-patient PSG was not always possible and as such, alternatives (home TOSCA or Oximetry) were employed where possible.

Overall the average age at the completion of palate repair was 9.2 months. This reflects our current approach to cleft repair. We work within a set of nationally agreed guidelines that are based on the Oslo approach to timing and ordering of cleft repair. Lips are repaired with the anterior palate using a vomerine flap between the ages of three and six months. Palate repairs are not undertaken before six months of age and should be complete by the age of thirteen months.

\section{Figure 2. Distribution of Desaturation Indices by Cleft Type}

Figures $2 a$. and $2 b$. represent the distribution of data obtained from preoperative and postoperative studies respectively. Data are presented as percentages of total number in each cleft presentation to allow direct comparison of cleft types. Data from sleep studies from 2 preoperative and 2 postoperative sleep studies from syndromic patients with isolated cleft palate have been excluded.

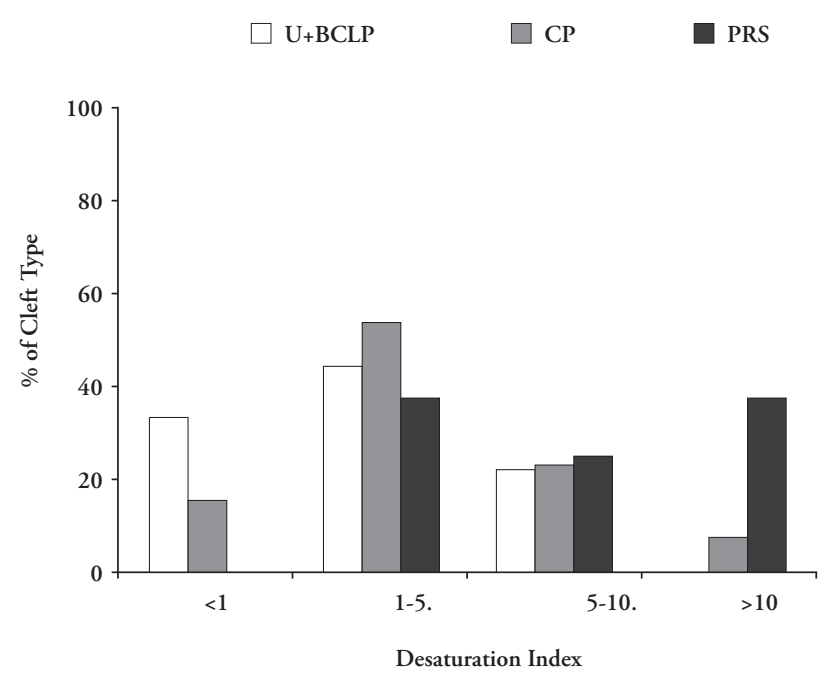

Figure 2a. Distribution of data from preoperative studies

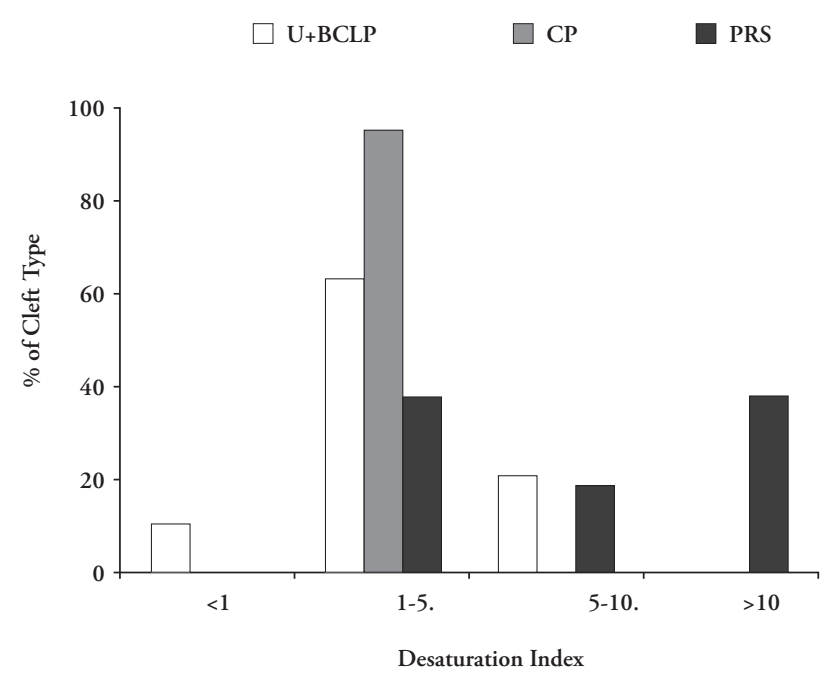

Figure 2b. Distribution of data from postoperative studies

We currently recruit to the Timing of Primary Surgery for Cleft Palate (TOPS) trial, which compares the outcome of cleft palate repair at either six or twelve months. The fact that our average age of repair between these two ages reflects well both the randomisation process within the trial and our equipoise as to the questions posed by the trial itself for those patients who have not consented to trial inclusion. 
PSG is the gold standard investigation of breathing during sleep. The Apnoea / Hypopnoea Index (AHI) output from PSG studies could be considered the gold standard measure of the severity of SDB as it differentiates central and obstructive events. However in this study, Desaturation Index have been used as it was the only relevant measure captured accurately by each modality of monitor used. Although the TOSCA and Masimo ${ }^{\circ}$ overnight oximetry studies are unable to differential central and obstructive events we did find that in those patients that had PSGs, most events were identified as obstructive, hence our use of airway adjuncts (NPA or Tracheostomy) rather than supplemental oxygen. The distribution of pre/post-operative SDB patterns demonstrated in Figure 2 raises the possibility that cleft repair itself might improve the situation. One explanation may be that the palatal repair prevents sub-luxation of the tongue into the cleft and thereby may result in improved nasal airflow and breathing. Alternatively, the improvements seen may be due to increase in general size of the children concerned as on average there was at least five months between the pre and post-operative study. Another factor may be maturation of control of upper airway muscle function. The fact that PRS patients did not demonstrate similar minor improvement in severity of SDB suggests a more proximal site of obstruction that is not overcome during the first year of their life.

As a result of our previous preconceptions we excluded cleft lip only patients from investigation. However currently with increasing experience in this area we have also found significant sleep disordered breathing patterns in this group. Therefore, given our findings and those of Maclean et al. (2009) ${ }^{4}$, we routinely perform pre-operative sleep studies on all our cleft patients and would recommend this practice. Furthermore as we have been unable to predict which patients have persisting evidence of SDB we would also recommend post-operative studies to guide continuing management. Serial sleep studies may be used to indicate safer operating windows and support informed consent by highlighting patients likely to require post-operative respiratory support.

\section{CONCLUSION}

Our experience is consistent with a Royal College of Paediatric Child Health report on disordered sleep physiology, which recommends sleep study evaluation on all patients with craniofacial malformation. ${ }^{10}$ This report identifies PRS patients in addition to cranio-facial patients as a cohort who would benefit from sleep investigations. Our data also suggest that these expensive but important investigations should not be restricted to PRS cleft patients but should be utilized in all cleft patients, as sleep disordered breathing can present in patients of all cleft types with varying significance.

At this time there are no available studies that indicate the long-term effects of SDB in cleft infants or normative data for comparison. Furthermore little or no evidence is available from appropriate cohorts to guide current clinical management. As such studies investigating this area are urgently required and need to be supported by research groups and clinical services.

\section{Acknowledgements:}

On Behalf of the West of Scotland Cleft Service, Royal Hospital for Sick Children, Yorkhill, Glasgow.

The Authors would like to thank Dr Andrew Morley and the rest of the Sleep Physiology Laboratory at the Royal Hospital for Sick Children for facilitating and performing the sleep investigations reported in this manuscript.

\section{REFERENCES}

1. Mossey P, Castillia E. Global registry and database on craniofacial anomalies: Report of a WHO Registry Meeting on Craniofacial Anomalies. Geneva: World Health Organization; 2003.

2. Mossey P, Little J, Munger RG, Dixon MJ, Shaw WC. Cleft lip and palate Lancet. 2009;374:1773-85.

3. Calzolari E, Pierini A, Astolfi G, Bianchi F, Neville AJ, Riveri F. Associated anomalies in multi-malformed infants with cleft lip and palate: an epidemiologic study of nearly 6 million births in 23 EUROCAT registries. Am J Med Genet. 2007;143:528-37.

4. MacLean JE, Hayward P, Fitzgerald DA, Waters K. Cleft lip and/or palate and breathing during sleep. Sleep Med Rev. 2009;13:345-54.

5. Gozal D, Crabtree VM, Sans CO, Witcher LA, KheirandishGozal L. C-reactive protein, obstructive sleep apnea and cognitive dysfunction in school-aged children. Am J Respir Crit Care Med. 2007; 176:188-93.

6. Gozal D. Sleep-disordered breathing and school performance in children. Paediatrics. 1998;102:616-20.

7. Liao YF, Noordhoff MS, Huang CS, Chen PK, Yun C, Chuang ML. Comparison of obstructive sleep apnea syndrome in children with cleft palate following Furlow palatoplasty or pharyngeal flap for velopharyngeal insufficiency. Cleft Palate Craniofac J. 2004;41(2):152-6.

8. Clinical Practice Guideline: diagnosis and management of childhood obstructive sleep apnoea syndrome. Pediatrics. 2002:109;704-12.

9. Brouillette RT, Morielli A, Leimanis A, Waters KA, Luciano R, Ducharme FM. Nocturnal Pulse Oximetry as an abbreviated testing modality for pediatric obstructive sleep apnoea. Pediatrics. 2000;105:405-12.

10. Royal College of Paediatric and Child Health Working Party on Sleep Physiology and Respiratory Control Disorders in Children [Internet]. Standards for Services for Children with Disorders of Sleep Physiology. [cited 2013 Oct 27]. Available from: http://www. rcpch.ac.uk/sites/default/files/asset_library/Research/Clinical\%20 Effectiveness/Endorsed\%20guidelines/Sleep\%20Physiology\%20 Disorders\%20\%28RCPCH\%29/Report\%20TextC.pdf 